TITLE:

\title{
Elucidation of conformational hysteresis on a giant DNA
}

$\operatorname{AUTHOR}(\mathrm{S})$ :

Shew, Chwen-Yang; Higuchi, Yuji; Yoshikawa, Kenichi

\section{CITATION:}

Shew, Chwen-Yang ...[et al]. Elucidation of conformational hysteresis on a giant DNA. Journal of Chemical Physics 2007, 127: 085103.

\section{ISSUE DATE:}

2007-08-28

URL:

http://hdl.handle.net/2433/45695

\section{RIGHT:}

Copyright (2007) American Institute of Physics. This article may be downloaded for personal use only. Any other use requires prior permission of the author and the American Institute of Physics. 


\title{
Elucidation of conformational hysteresis on a giant DNA
}

\author{
Chwen-Yang Shew ${ }^{\text {a) }}$ \\ Department of Chemistry, College of Staten Island and Graduate Center, City University of New York, 2800 \\ Victory Boulevard, Staten Island, New York 10314, USA \\ Yuji Higuchi and Kenichi Yoshikawa \\ Department of Physics, Graduate School of Science, Kyoto University, Kyoto 606-8502, Japan
}

(Received 22 February 2007; accepted 25 June 2007; published online 28 August 2007)

\begin{abstract}
The conformational behavior of a giant DNA mediated by condensing agents in the bulk solution has been investigated through experimental and theoretical approaches. Experimentally, a pronounced conformational hysteresis is observed for folding and unfolding processes, by increasing and decreasing the concentration of condensing agent (polyethylene glycol) (PEG), respectively. To elucidate the observed hysteresis, a semiflexible chain model is studied by using Monte Carlo simulations for the coil-globule transition. In the simulations, the hysteresis loop emerges for stiff enough chains, indicating distinct pathways for folding and unfolding processes. Also, our results show that globular state is thermodynamically more stable than coiled state in the hysteresis loop. Our findings suggest that increasing chain stiffness may reduce the chain conformations relevant to the folding pathway, which impedes the folding process. () 2007 American Institute of Physics. [DOI: 10.1063/1.2759925]
\end{abstract}

\section{INTRODUCTION}

The conformational behavior of a giant DNA has been actively studied over the past few years because its unique morphology is relevant to the fundamental physics of life science. ${ }^{1-4}$ For example, the experimental evidence has linked the conformational transition of a long chain DNA with the switching of transcriptional activities on-and-off in $v_{i v o}{ }^{2}$ and with the rate of DNA polymerase. ${ }^{5}$ It is known that elongation of a compact DNA is important to facilitate the above cellular activities in the presence of cellular proteins. ${ }^{1,2,5}$ The cellular proteins play the role on altering the media condition surrounding a DNA molecule. Such a system is similar to the fundamental coil-to-globule conformational transition in polymer physics. ${ }^{6}$

Experimentally, the coiled and globular DNAs can be induced by changing the amount of condensing agent, for instance, high valent cations or neutral polyethylene glycol (PEG) etc. More than a decade ago, by using the single molecular fluorescence image, it has been found that the coilto-globule transition of DNA is of the first-order (all-ornone) phase transition with coexistent coiled and globular conformational states. ${ }^{1,2}$ The change in the effective volume is of the order of $10^{4}-10^{5} .^{1}$ In the discrete conformational transition, there exists a region of coexistent elongated and compact DNA chains. Since the effective segmental density in the elongated state is very low compared to that in the compact state, the precise intensity on the light scattering is very weak. This means that the physicochemical properties of the ensemble of chains, such as light scattering of a DNA solution, seem continuous. ${ }^{1}$ To this end, the single molecular fluorescence image remains the most suitable experimental method for detecting single chain conformations.

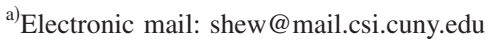

When the concentration of condensing agents is not in large excess, the compact DNA chains are soluble and do not aggregate with each other even through frequent collisions in solution. Under this condition, the kinetic processes of nucleation and growth can also be observed. ${ }^{7}$ Through such processes, a regular toroidal structure is generated as the product of chain compaction. However, when a large excess of condensing agent is added to the DNA solution, a rapid collapse is induced by the formation of a spherical product via irregular packing of the chain. ${ }^{8}$ Such behavior of single DNA molecules is similar to the kinetic aspects of crystallization from a supercooled solution: when the degree of supercooling is small, a regular pure crystal is obtained; on the other hand, when the degree is large, a "dirty" precipitate is obtained (spinodal decomposition). In compact particles with regular tight packing, such as a toroid and rod, the negative charge disappears completely except on the surface. ${ }^{9}$ The surviving negative charge on the surface of the compact DNA enhances its soluble colloid properties.

From the theoretical standpoint, the unique conformational behavior of a giant DNA chain has been mainly attributed to its semiflexibility. ${ }^{1,8}$ The simulation results have revealed that the coil-to-globule transition is continuous for flexible chains but is discontinuous for stiff chains such as DNA. ${ }^{8}$ Also, unlike the liquid-like globule for a collapsed flexible chain, an ordered (crystal-like) toroid structure can be formed in the compact state of DNA. ${ }^{4,10}$ These findings are consistent with experimental observations. ${ }^{1,3,8}$

In addition to characterization of the biphasic DNA conformations, the dynamics involved in the conformational transition has also attracted a great deal of attention. The techniques by using extensional force and extensional flow methods have been developed to stretch a DNA chain. ${ }^{11-14}$ The hysteresis loop was identified in the stretching and re- 
TABLE I. Summary of solution preparation for forward and backward titration. Concentrations of stock solutions: $[\mathrm{NaCl}]=2 \mathrm{M}$ (from Nacalai Tesque), $[\mathrm{PEG}]=200 \mathrm{mg} / \mathrm{ml}$ (molecular weight of $20000 \mathrm{D}$ from Kishida Chemical, Osaka), [DAPI] $=10 \mu \mathrm{M}$, and [DNA] $=20 \mu \mathrm{M}$ in base units (bacteriophage T4DNA, $166 \mathrm{kbps}$, from Nippon Gene).

\begin{tabular}{cccc}
\hline \hline Solution & $\begin{array}{c}\text { Forward titration } \\
\text { amount used }(\mu \mathrm{l})\end{array}$ & $\begin{array}{c}\text { Backward titration (a) } \\
\text { amount used }(\mu \mathrm{l})\end{array}$ & $\begin{array}{c}\text { Backward titration }(\mathrm{b}) \\
\text { amount used in titrant }(\mu \mathrm{l})\end{array}$ \\
\hline De-ionized water & $630-505$ & $540-535$ & $820-760$ \\
NaCl solution & 150 & 150 & 150 \\
PEG solution & $200-325$ & 300 & $\ldots$ \\
DAPI solution & 10 & $\ldots$ & $30-90$ \\
DNA solution & 10 & around $10-15$ & $\ldots$ \\
Final solution & 1000 & 1000 & 1000 \\
\hline \hline
\end{tabular}

leasing processes of these experiments, suggesting that the long chain DNA undertakes different pathways for the above two processes. Nevertheless, in these experiments, the conformational transition was induced by external perturbations. Hence, the experiment to elucidate the solvent-induced conformational transition is greatly needed. Such an experiment provides insights into the thermodynamics and kinetics of the DNA coil-to-globule transition and the molecular machinery of life. ${ }^{15}$

Despite the prediction from simulations, ${ }^{10}$ to the best of our knowledge, the direct measurement for the forward and reverse transitions between coiled and globular states has not been reported. In this work, we intend to elucidate the conformational hysteresis of a giant DNA mediated by condensing agents via integrating experimental and theoretical methods. In the experiment, PEG will be introduced as condensing agent, and both titration and back titration will be conducted to investigate the folding and unfolding processes of DNA. Meanwhile, a simplified model will be employed to study the folding and unfolding processes through dynamic Monte Carlo simulation suggested by Milchev et al. $^{16}$ The density-of-state Monte Carlo method ${ }^{17}$ will be further applied to determine the stable conformational state in the hysteresis loop.

\section{EXPERIMENT}

Single chain observation on individual giant DNA molecules by use of fluorescence microscopy has been performed following the procedures, as described in our previous reports. ${ }^{18,19}$ This method renders an opportunity to provide precise measurement for the chain conformation of free DNA molecules in the bulk solution. Thus, in this section, we will mainly describe the experimental method exploited to elucidate the hysteresis on the conformational transition of DNA molecules.

In the experiment, the titration and back titration of bacteriophage T4DNA [166 kilo base pairs (kbps)] solutions with PEG (molecular weight of $20000 \mathrm{D})$ were carried out to investigate the conformational behavior of a giant DNA molecule in the presence of condensing agents. The details are summarized in Table I. The required substances were mixed based on the order in the table, from top (water) to bottom (DNA). In the forward titration, a series of PEG solutions were prepared through adjusting the quantity of water (between 630 and $505 \mu \mathrm{l}$ ) and PEG (between 200 and $325 \mu \mathrm{l}$ of stock solution), but keeping the final solution to be $1000 \mu \mathrm{l}$. The resulting solutions contain $0.2 \mu \mathrm{M}$ (in base units) of DNA in $300 \mathrm{mM} \mathrm{NaCl}$ with $0.1 \mu \mathrm{M}$ of fluorescent dye 4', 6-diamidino-2-phenylindole, (DAPI), and the PEG concentration was increased from 40 to $65 \mathrm{mg} / \mathrm{ml}$ in the forward titration.

Prior to back titration, two sets of solutions were made separately according to the quantity and the mixing order (from top to bottom) listed in the third and fourth columns of Table I. The first set of solutions [solution (a) in Table I] contained DNA of different concentrations (10-15 $\mu \mathrm{l}$ of DNA stock solution mixed with $\mathrm{NaCl}$ and PEG solutions), and each final solution was diluted to $1000 \mu \mathrm{l}$. These solutions were under incubation at $21^{\circ} \mathrm{C}$ for $60 \mathrm{~min}$ to collapse all DNA molecules into compact state. In the second set of solutions [solution (b) in Table I], different amounts of water (820-760 $\mu \mathrm{l})$ and DAPI (30-90 $\mu \mathrm{l}$ of stock solution) were mixed with $150 \mu \mathrm{l}$ of $\mathrm{NaCl}$ stock solution. Each solution was diluted to $1000 \mu \mathrm{l}$. In the back titration, the DNA and titrant solutions [i.e., solutions (a) and (b)] were mixed by using the proportions given in Table II to keep the DNA concentration around $0.2 \mu \mathrm{M}$. Table II also manifests the concentrations of various substances for each final solution. In the backward titration, the PEG concentration was decreased from 54 to $33 \mathrm{mg} / \mathrm{ml}$.

TABLE II. Solution preparation of substance concentrations for backward titration. The final solution for back titration was obtained by mixing solutions (a) and (b) in Table I.

\begin{tabular}{ccccc}
\hline \hline Solution (a):(b) & $\mathrm{NaCl}(\mathrm{mM})$ & PEG $(\mathrm{mg} / \mathrm{ml})$ & DAPI $(\mu \mathrm{M})$ & DNA $(\mu \mathrm{M})$ \\
\hline $5.5: 4.5$ & 300 & 33 & 0.090 & 0.17 \\
$6.0: 4.0$ & 300 & 36 & 0.12 & 0.18 \\
$6.5: 3.5$ & 300 & 39 & 0.11 & 0.20 \\
$7.0: 3.0$ & 300 & 42 & 0.12 & 0.21 \\
$7.5: 2.5$ & 300 & 45 & 0.10 & 0.18 \\
$8.0: 2.0$ & 300 & 48 & 0.10 & 0.19 \\
$8.5: 1.5$ & 300 & 51 & 0.11 & 0.20 \\
$9.0: 1.0$ & 300 & 54 & 0.081 & 0.18 \\
\hline \hline
\end{tabular}


For both forward and backward titrations, each final solution was incubated for 60 and $1000 \mathrm{~min}$ at $21^{\circ} \mathrm{C}$. After incubation, the single molecular fluorescence images were applied to count the number of coiled and compact DNA molecules in solution at the same temperature. To determine the fractions of coiled and globular states, a total of 100-270 DNA molecules were examined. The counting was repeated two to five times for each titration point, and the standard deviation was calculated.

To obtain single molecular conformations without interference from intermolecular interaction, very dilute DNA solutions were prepared, around $0.2 \mu \mathrm{M}$. It has been reported that the radius of gyration of the coiled T4DNA (166 kbps) is $1.4 \mu \mathrm{m},{ }^{20}$ and the corresponding overlap concentration is estimated to be $200 \mu \mathrm{M}\left(\approx 1 / R_{g}^{3}\right.$, where $R_{g}$ is the radius of gyration). ${ }^{21}$ Our experimental procedures assure that the DNA solutions are well below the DNA overlap concentration for both coiled and globular states. Also, the thus obtained chain conformations are independent of experimental DNA concentrations, which have been confirmed in the previous work. ${ }^{22}$ Besides, the $\mathrm{NaCl}$ concentration $(300 \mathrm{mM})$ remained the same for the entire hysteresis experiment. The high $\mathrm{NaCl}$ concentration chosen in this work is to screen out the charges of a giant DNA and allows us to examine the effect of PEG on the semiflexible DNA conformation alone.

\section{MODEL AND MONTE CARLO SIMULATIONS}

To abstract the essential mechanism on the observed hysteresis, Monte Carlo simulations are resorted. In the simulations, an isolated finite extension nonlinear elastic chain (with eight monomers and thirty-two monomers) is considered, consisting of bond vibrational energy, repulsive Lennard-Jones (LJ) potential, and bending energy. ${ }^{23}$ The bending energy adjusts the chain stiffness, which reads $\kappa$ $\left(\theta-\theta_{0}\right)$, where $\kappa$ is the strength of bending energy, $\theta$ is the bond angle, and $\theta_{0}=\pi$. The parameters of these potentials are obtained from Ref. 23. To mimic the poor solvent condition arising from condensing agents, an effective attractive interaction between any two monomers is incorporated, which takes the form of Yukawa potential, given by $V_{\text {att }}=$ $-\epsilon \exp (-B r) / r$, where $\epsilon$ is the strength of attractive interaction and $B^{-1}$ is the characteristic length of the attractive interaction. In the calculations, the LJ diameter, the Boltzmann constant, and temperature are assumed to be unity, and $B=1$.

Monte Carlo simulations are carried out by using the traditional Metropolis and density-of-states (DOS) methods. In the Metropolis Monte Carlo simulations, the chain conformation is relaxed by using random walk for monomers, equivalent to the Rouse motion of a polymer chain as suggested by Milchev et al., ${ }^{16}$ and a total of $1 \times 10^{8}$ moves for $N=8$ and $2 \times 10^{8}$ moves for $N=32$ are carried out for each case. In the DOS Monte Carlo method, we follow the algorithm suggested by Wang and Landau to compute the density of states $\Omega(E),{ }^{17}$ and the simulation is mainly conducted for shorter chains that give better statistics. (a)

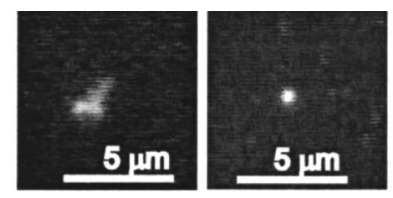

(b)
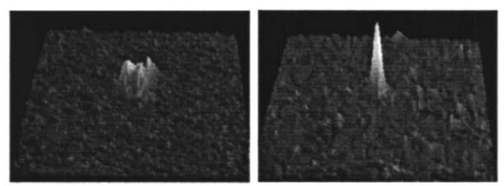

(c)

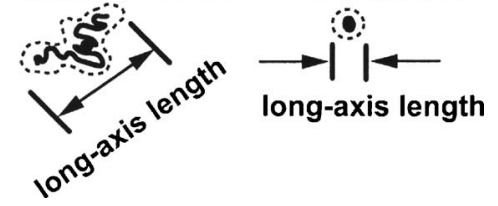

FIG. 1. Fluorescence images of a single T4DNA existing in the bulk solution with two distinct conformations in (a) (elongated chain on left and compact chain on right), the quasi-3D representation corresponding to the light intensity distribution in the fluorescence image in (b), and the schematic of the long-axis length $L$, which is defined as the longest distance in the outline of DNA images in (c).

\section{RESULTS AND DISCUSSION}

The conformational behavior of a giant DNA in the bulk solution is characterized by using single molecular images. Figure 1 displays the fluorescence images of a single T4DNA for two distinct conformations (elongated chain on left and compact chain on right) in (a), the quasi-threedimensional (3D) representation corresponding to the light intensity distribution in the fluorescence image in (b), and the schematic of the long-axis length $L$, which is defined as the longest distance in the outline of DNA images along with the blurred effect, $0.3 \mu \mathrm{m}$, in (c). The conformations in Fig. 1(a) are two typical chain morphologies for coiled and globular states, respectively, with very different volumes. The structure of each conformation is further verified through the quasi-3D representation in Fig. 1(b). From these measurements, the chain size is determined based on the long-axis length $L$, which serves as an order parameter in the analysis of chain size distribution.

In relation to them, it has been confirmed in the previous studies that the bimodal distribution around the transition region is well characterized by the measurement of long-axis length, as well as the other measurements such as hydrodynamic radius $R_{h}$ and radius of gyration $R_{g} .{ }^{24}$ It is to be noted that we have performed fluorescence microscopy observation on the DNA molecules existing exclusively in the bulk solution. The low-resolution images are unavoidable for such a measurement. This is because the Brownian motion of the DNA in the bulk solution causes the blurring effect on the images. Nevertheless, the blurring effect $(\sim 0.3 \mu \mathrm{m})$ is smaller than the chain size (long DNA chains used in our experiment), and the measured conformation of single DNA molecules yields the precise information on the conformational transition. In contrast, the DNA absorbed onto the glass plate exhibits a much better resolution than those in bulk solutions. Through image resolutions, the chain molecules under Brownian motion in the bulk solution and those 


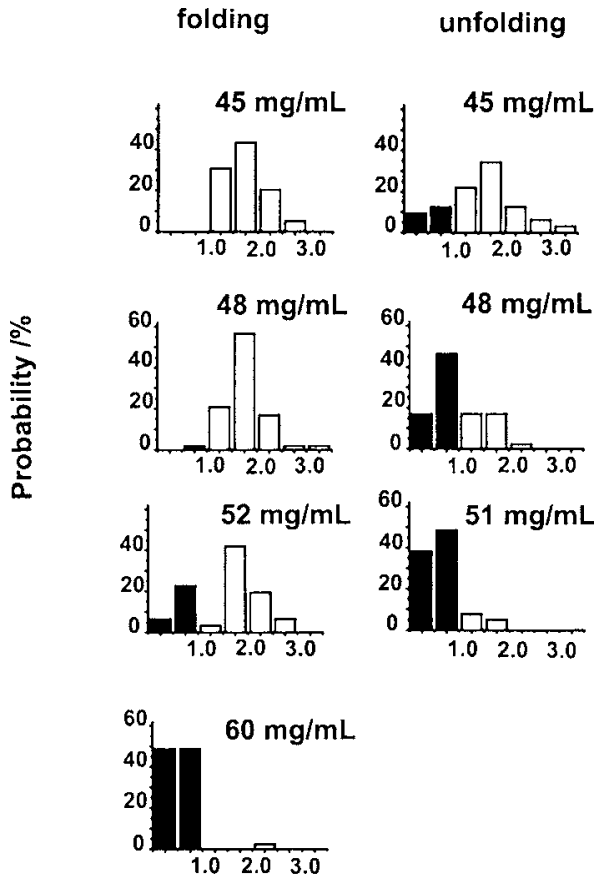

unfolding

Long-axis Length, $L / \mu \mathrm{m}$

FIG. 2. Plot of the histograms of the long-axis length $L$ of T4DNA molecules with an increase of PEG concentration during forward titration from top to bottom $(45,48,52$, and $60 \mathrm{mg} / \mathrm{ml})$ on left column and with a decrease of PEG concentration during back titration from bottom to top (51, 48 , and $45 \mathrm{mg} / \mathrm{ml}$ ) on right column after incubation of $60 \mathrm{~min}$ for each concentration. All of the data were obtained from the DNA molecules existing in the bulk solution.

adsorbed onto the surface can be distinguished. Thus, in this manuscript, only the data of the DNA molecules in the bulk solution are shown.

To examine the conformational behavior along the forward and backward titrations, the chain size distribution is investigated. Figure 2 plots the histograms of the long-axis length $L$ of T4DNA molecules with an increase of PEG concentration during forward titration from top to bottom (45, 48,52 , and $60 \mathrm{mg} / \mathrm{ml}$ ) on left column and with a decrease of PEG concentration during back titration from bottom to top $(51,48$, and $45 \mathrm{mg} / \mathrm{ml})$ on right column after incubation of $60 \mathrm{~min}$ for each concentration. In forward titration, the chain size distribution for low PEG concentrations is basically single modal, and the peak is located at a larger $L(\approx 1.5 \mu \mathrm{m})$. As PEG concentration is increased to $48 \mathrm{mg} / \mathrm{ml}$, the bimodal distribution starts to emerge. When PEG concentration is increased further to $52 \mathrm{mg} / \mathrm{ml}$, the bimodal distribution becomes more pronounced, suggesting that DNA molecules fold and undergo a discontinuous transition with coexistent coiled and globular conformational states. At a high PEG concentration $(60 \mathrm{mg} / \mathrm{ml})$, the distribution is single modal, and the peak position shifts toward a smaller $L, \sim 0.5 \mu \mathrm{m}$ for mean $L$. The result indicates that all DNA molecules are transformed from coiled state at a low PEG concentration $(45 \mathrm{mg} / \mathrm{ml})$ to globular state at a high PEG concentration $(60 \mathrm{mg} / \mathrm{ml})$ through the transition, as seen in the first-order phase transition.

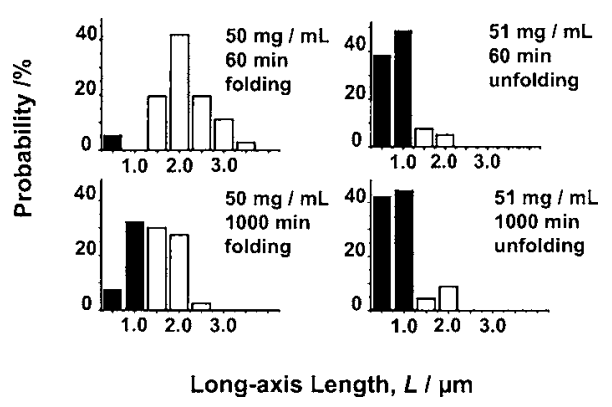

FIG. 3. Plots of the histograms of DNA chain size in the bulk solution at $[\mathrm{PEG}]=50 \mathrm{mg} / \mathrm{ml}$ during the titration process (on left) and at [PEG] $=51 \mathrm{mg} / \mathrm{ml}$ during the back titration process (on right) for different incubation times, 60 (top) and $1000 \mathrm{~min}$ (bottom).

In back titration, the qualitative features are reversed in which more DNA molecules unfold as the PEG concentration is decreased. Nevertheless, the quantitative difference between folding (titration) and unfolding (back titration) processes can be discerned for [PEG] $=45$ and $48 \mathrm{mg} / \mathrm{ml}$ after 60 min incubation, for example. In these PEG concentrations, the profiles of long-axis $L$ distribution of forward titration (folding) are significantly different from those of back titration (unfolding). The statistical weight of globular state remains higher for the back titration (unfolding) process.

In addition to the amount of condensing agent, it is noted that the statistical distribution of coiled and globular states is also dependent on the processes of titration and/or incubation time. In the following, the investigation is conducted during forward titration and back titration processes. For each process, one PEG concentration is selected to study the effect of incubation time. Figure 3 displays the histograms of DNA chain size at $[\mathrm{PEG}]=50 \mathrm{mg} / \mathrm{ml}$ during the forward titration process (on left) and at $[\mathrm{PEG}]=51 \mathrm{mg} / \mathrm{ml}$ during the back titration process (on right) for different incubation times, 60 (top) and $1000 \mathrm{~min}$ (bottom). In the forward titration process, the probability of finding globular conformations is low for the 60 min incubation time. After increasing the incubation time to $1000 \mathrm{~min}$, the bimodal profile of the chain size distribution becomes more pronounced with an increase of globular chains in solution. On the contrary, in the case of back titration, the measured histogram is not sensitive to incubation time. The results may be attributed to different conformational behaviors in the titration and back titration processes.

The above analysis based on histograms allows us to estimate the mean statistical weight of the two coexistent conformational states during the forward titration and back titration processes. Figure 4 displays the fraction of elongated (or coiled) state $f_{\text {coil }}$ obtained from forward titration and back titration, denoted by solid and open symbols, respectively, after incubation for $60(\bigcirc)$ and $1000 \mathrm{~min}(\Delta)$; lines provide a visual guide. It is noticeable that besides experimental uncertainty, the chain size distribution is the other cause to broaden the error bars in Fig. 4, particularly near the transition point with large chain size fluctuation. In the forward titration, $f_{\text {coil }}$ decreases as PEG concentration is increased (facilitating chain folding) for a given incubation time. Furthermore, $f_{\text {coil }}$ decreases with an increase of incuba- 


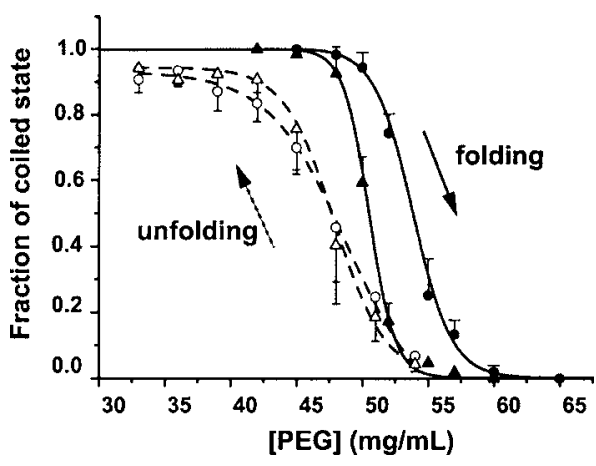

FIG. 4. Variation of the fraction of elongated chains $f_{\text {coil }}$ in the bulk solution with PEG concentration obtained from (forward) titration and back titration, denoted by solid and open symbols, respectively, after incubation for $60(\bigcirc)$ and $1000 \min (\triangle)$ (lines provide a visual guide.) Note that only a half of an error bar is sketched, and the magnitude of error bars arises from both experimental uncertainty and chain size distribution.

tion time from 60 to $1000 \mathrm{~min}$, and the titration curve shifts to smaller $f_{\text {coil }}$. Unlike forward titration, the result of back titration exhibits a different behavior. As PEG concentration is decreased in the back titration, fewer DNA chains tend to elongate; consequently, the number of elongated chains is smaller compared to the forward titration at a given PEG concentration. It is noticeable that for the unfolding process, $f_{\text {coil }}$ is dependent on the PEG concentration but becomes less sensitive to incubation time. The conformational hysteresis in the titration loop indicates different pathways involved in the folding and unfolding processes of a giant DNA molecule in the presence of condensing agents. Note that some DNA molecules remain the compact form in the dilute PEG solution even for the longest incubation time under the unfolding process. In general, nucleation occurs in the first-order phase transition. ${ }^{25}$ Since the DNA conformational transition mediated by condensing agents is the first-order transition, the slow nucleation process is expected for chain folding (but not for the unfolding process). ${ }^{7,26,27}$ Hence, the folding process is slower than the unfolding process from the standpoint of kinetics.

To elucidate the conformational hysteresis, the simplified semiflexible chain model introduced in Sec. III is studied. The mean chain size is first calculated through the randomwalk Monte Carlo method in the following way. In the folding process, the simulation begins with the coil state, and then the monomer-monomer attraction $\epsilon$ is increased gradually until the chain collapses into compact state. After the chain collapses totally, the reverse process is applied to unfold the chain by decreasing $\epsilon$, and $\epsilon$ is decreased until $\epsilon=0$. Figure 5 plots the variation of mean squared radius of gyration, divided by a factor $\Gamma$, with $\epsilon$ and some snapshots for $\kappa=2(\Gamma=1)$ and $10(\Gamma=10)$ when $N=8$, and for $\kappa=20$ ( $\Gamma=100$ ) when $N=32$, as marked, for the folding (solid symbols) and unfolding (open symbols) processes; lines provide a visual guide. The factor $\Gamma$ is introduced to collapse all the curves in the same plot. As the monomer-monomer attraction is increased, the chain starts to contract. For a more flexible chain (e.g., $\kappa=2$ and $N=8$ ), the transition between an elongated and a compact state is reversible. As the chain stiffness is increased (e.g., $\kappa=10$ and $N=8$ or $\kappa=20$ and $N=32$ ), a

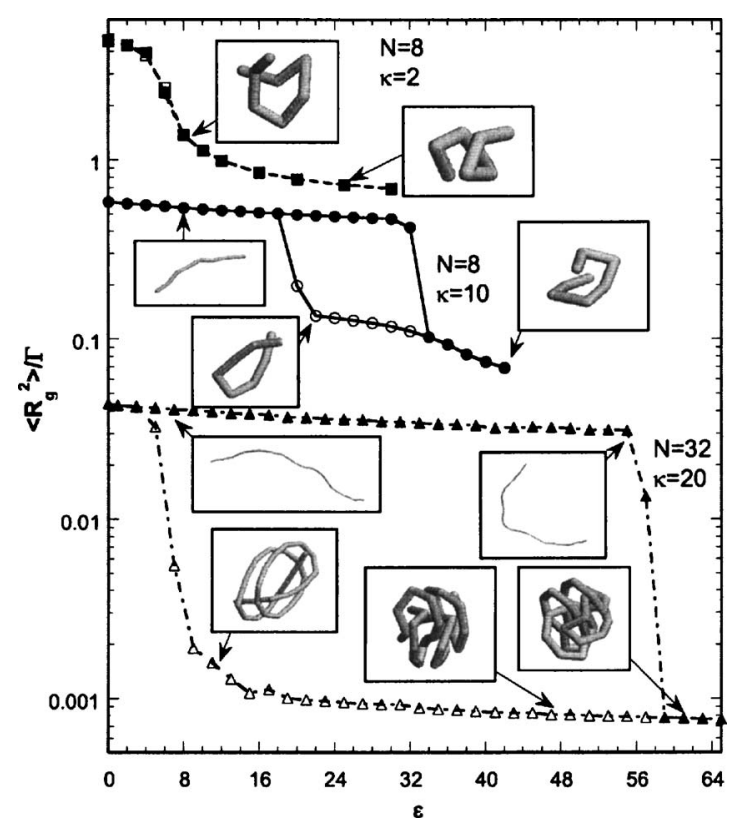

FIG. 5. Variation of mean squared radius of gyration, divided by a factor $\Gamma$, with $\epsilon$ and some snapshots for $\kappa=2(\Gamma=1)$ and $10(\Gamma=10)$ when $N=8$ and for $\kappa=20(\Gamma=100)$ when $N=32$, as marked, under the folding (solid symbols) and unfolding (open symbols) processes; lines provide a visual guide, and the factor $\Gamma$ is introduced to collapse all the curves in the same plot.

conformational hysteresis occurs, indicating distinct folding and unfolding pathways for both processes. Besides, we find that once the chain collapses, unfolding the chain (particularly for longer chains, e.g., $N=32$ ) back to its elongated state becomes difficult. These results are consistent with the experimental observations in Fig. 4.

The simulation snapshots exhibit the chain structures during the folding and unfolding processes. In Fig. 5, for the less stiff chain, such as $\kappa=2$ and $N=8$, the folding and unfolding processes are reversible, and the chain tends to form cyclic conformation after it collapses, e.g., $\epsilon=8$. A further increase of $\epsilon$ forces the chain to contract more, e.g., $\epsilon=26$. For a stiffer chain, such as $\kappa=10$ and $N=8$, the polymer collapses from an elongated chain (e.g., $\epsilon=8)$ to a compact ring structure (e.g., $\epsilon=42$ ) in the folding process. During the unfolding process, the compact chain takes a more expanded ring structure (e.g., $\epsilon=22$ ) before it becomes totally elongated. For a longer and stiffer chain, e.g., $\kappa=20$ and $N=32$, the elongated chain conformation persists until $\epsilon \approx 56$, and the collapsed chain adopts a highly compact structure consisting of four smaller rings intercalating with each other, as shown in $\epsilon=61$. In the unfolding process, the chain makes a transition from the compact structure (e.g., $\epsilon=47$ similar to that of $\epsilon=61)$ to two rings (e.g., $\epsilon=11)$ before it is totally elongated. These results again show the distinct pathways for folding and unfolding processes.

A further calculation is conducted to identify the stable conformational state in the hysteresis loop. Figure 6 compares the energy distribution (divided by a factor $\gamma$ ) of the elongated and compact states obtained from the Metropolis method (solid lines and $\gamma=1$ ) with that of the DOS method (dotted line and $\gamma=2$ ) for $\kappa=10$ and $\epsilon=30$ when $N=8$. The result shows that the DOS method, independent of the choice 


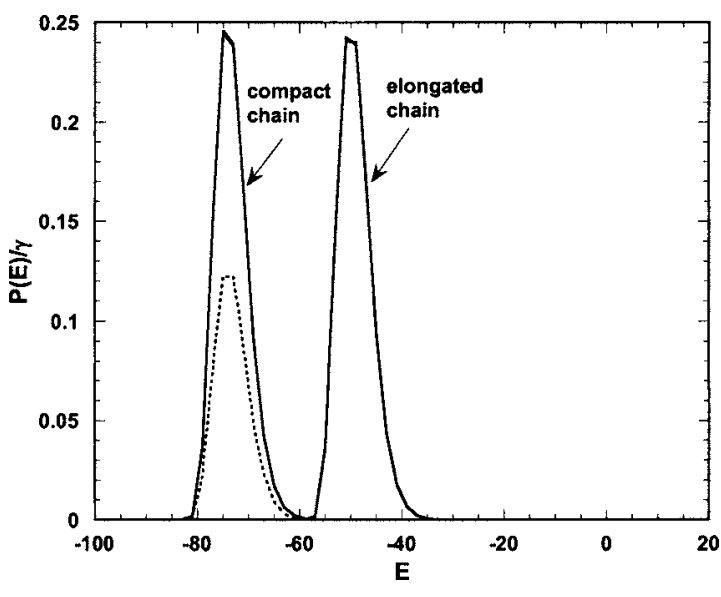

FIG. 6. Comparison of the energy distribution (divided by a factor $\gamma$ ) of the elongated and compact states obtained from the Metropolis method (solid lines, $\gamma=1$ ) with that of the DOS method (dotted line, $\gamma=2$ ) for $\kappa=10$ and $\epsilon=30$ when $N=8 ; \gamma$ is introduced to differentiate the results from the two different methods.

of initial conformations, agrees well with the energy distribution of the compact state in the hysteresis, suggesting that the compact state is thermodynamically more stable and the elongated state is a metastable state. The folding process is impeded during the length of our simulations, probably due to the energy trapping for a polymer chain.

From the simulations, the effect of chain stiffness on the density of states is examined. Figure 7 plots the renormalized density of states $\Omega(E)$ for $\kappa=5$ and 10 , as marked, and for $\epsilon=0$ (solid lines) and 4 (dotted lines) when $N=8$; the straight line denotes the Boltzmann factor. The crossing point between the line of the Boltzmann factor and each curve reflects the rough peak position of the energy distribution function for a given set of $\kappa$ and $\epsilon$. In contrast to a more flexible chain, the energy distribution of a stiffer chain appears at a higher energy, and the value of its density of states is smaller at around the line equivalent to Boltzmann factor. As the monomer-monomer attraction is increased, the energy distribution shifts to a lower energy, but the density of states of a stiffer chain remains smaller than that of a flexible chain.

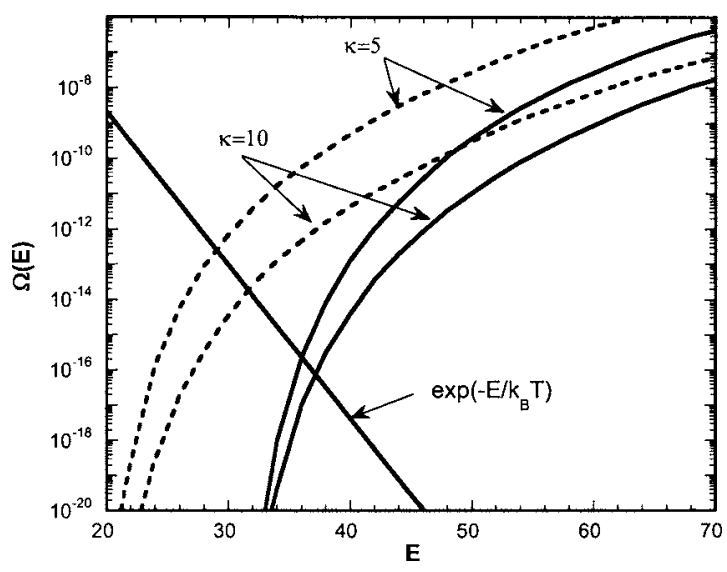

FIG. 7. Plot of the renormalized density of states for $\kappa=5$ and 10 , as marked, and for $\epsilon=0$ (solid lines) and 4 (dotted lines) when $N=8$; the straight line denotes the Boltzmann factor.
The different kinetic pathways for the folding and unfolding processes of a giant DNA have been addressed extensively in literature. ${ }^{7,26,27}$ The slow nucleation is the first step of the folding process as in the intramolecular nucleation involved in polymer crystallization ${ }^{28,29}$ but not for the unfolding process. ${ }^{7,26,27}$ An increase of the attraction between monomers stabilizes the nucleation site and facilitates the subsequent growth of the folded structure. In this work, a complementary explanation from thermodynamic standpoint is given as follows. A decrease of the density of states by increasing chain stiffness is corresponding to attenuation of possible chain conformations in a semiflexible chain. We speculate that as the total number of conformations becomes low, those conformations relevant to the folding pathway may also decrease simultaneously. As a result, the folding process is impeded, in particular, if the folding related conformations bear higher energies. With an increase of monomer-monomer attraction, the density of states becomes larger, which increases the number of possible conformations as well as the probability of folding. Furthermore, the low density of states of a semiflexible chain sheds new light on its ordered compact structure. For a flexible chain polymer, the chain molecule collapses into a liquidlike globule, whereas for a semiflexible chain, it forms a highly ordered toroid. $^{1,8}$ Such a highly ordered structure in the compact globule state may link with the low density of states in a semiflexible DNA molecule.

\section{CONCLUSIONS}

The titration and back titration with PEG have been conducted to investigate the conformational behavior of a giant DNA molecule mediated by condensing agents in the bulk solution. A pronounced conformational hysteresis is observed for folding and unfolding processes, by increasing and decreasing PEG concentration, respectively, in experiment. To abstract the essential mechanism on the observed hysteresis, a semiflexible chain model is studied by using the random-walk Monte Carlo simulation to mimic the Brownian motion of monomers. The semiflexible chain molecule is modeled as a nonlinear elastic spring subjected to a bending energy. A monomer-monomer attractive interaction is incorporated to model poor solvent conditions. The simulation is conducted in the following way. The folding process is proceeded as the monomer-monomer attraction is increased, and after the chain is collapsed into the compact state, the unfolding process is followed by decreasing the monomermonomer attraction. As the chain becomes stiff enough, a conformational hysteresis occurs in the random-walk Monte Carlo simulations. The density-of-states Monte Carlo method shows that the compact state is thermodynamically more stable than the elongated one in the hysteresis loop. Our results suggest that the chain stiffness induced conformational hysteresis may reduce the chain conformations relevant to the folding pathway of a semiflexible chain. As a result, the folding process becomes thermodynamically unfavorable because of the low probability to reach the intermediate state. 


\section{ACKNOWLEDGMENTS}

One of the authors (C.Y.S.) received partial support for this work from the City University of New York PSC-CUNY grants, CSI Presidential Research Award, and NY STAR grant for the Center of Engineered Polymeric Materials at CSI and Institute of Macromolecular Assembly. This work was supported by Japan Society for the Promotion of Science (JSPS) under a Grant-in-Aid for Creative Scientific Research (Project No. 18GS0421).

${ }^{1}$ K. Yoshikawa, Adv. Drug Delivery Rev. 52, 235 (2001), and references therein.

${ }^{2}$ K. Yoshikawa, J. Biol. Phys. 31, 243 (2005), and references therein.

${ }^{3}$ V. A. Bloomfield, Curr. Opin. Struct. Biol. 6, 334 (1996).

${ }^{4}$ Y. Takenaka, K. Yoshikawa, Y. Yoshikawa, Y. Koyama, and T. Kanbe, J. Chem. Phys. 123, 014902 (2005).

${ }^{5}$ J. F. Leger, J. Robert, L. Bourdieu, D. Chatenay, and J. F. Marko, Proc. Natl. Acad. Sci. U.S.A. 95, 12295 (1998).

${ }^{6}$ I. M. Lifshitz, A. Y. Grosberg, and A. R. Khokhlov, Rev. Mod. Phys. 50, 683 (1978).

${ }^{7}$ K. Yoshikawa and Y. Matsuzawa, J. Am. Chem. Soc. 118, 929 (1996).

${ }^{8}$ H. Noguchi and K. Yoshikawa, J. Chem. Phys. 109, 5070 (1998).

${ }^{9}$ Y. Yamasaki, Y. Teramoto, and K. Yoshikawa, Biophys. J. 80, 2823 (2001).

${ }^{10}$ J. A. Martemyanova, M. R. Stukan, V. A. Ivanov, M. Muller, W. Paul, and K. Binder, J. Chem. Phys. 122, 174907 (2005).

${ }^{11}$ C. G. Baumann, V. A. Bloomfield, S. B. Smith, C. Bustamante, M. D.
Wang, and S. M. Block, Biophys. J. 78, 1965 (2000).

${ }^{12}$ Y. Murayama, Y. Sakamaki, and M. Sano, Phys. Rev. Lett. 90, 018102 (2003).

${ }^{13}$ S. A. Harris, Contemp. Phys. 45, 11 (2004).

${ }^{14}$ C. M. Schroeder, H. P. Babcock, E. S. G. Shaqfeh, and S. Chu, Science 301, 1515 (2003)

${ }^{15}$ K. Yoshikawa and H. Noguchi, Chem. Phys. Lett. 303, 10 (1999).

${ }^{16}$ A. Milchev, K. Binder, and A. Bhattacharya, J. Chem. Phys. 121, 6042 (2004).

${ }^{17}$ F. Wang and D. P. Landau, Phys. Rev. Lett. 86, 2050 (2001).

${ }^{18}$ K. Yoshikawa, M. Takahashi, V. V. Vasilevskaya, and A. R. Khokhlov, Phys. Rev. Lett. 76, 3029 (1996).

${ }^{19}$ H. Murayama and K. Yoshikawa, J. Phys. Chem. B 103, 10517 (1999).

${ }^{20}$ V. V. Vasilevskaya, A. R. Khokhlov, Y. Matsuzawa, and K. Yoshikawa, J. Chem. Phys. 102, 6595 (1995).

${ }^{21}$ M. Doi and S. Edwards, The Theory of Polymer Dynamics (Clarendon, Oxford, 1986).

${ }^{22}$ T. Iwataki, S. Kidoak, T. Sakaue, K. Yoshikawa, and S. S. Abramchuk J. Chem. Phys. 120, 4004 (2004).

${ }^{23}$ M. J. Stevens, Biophys. J. 80, 130 (2001).

${ }^{24}$ M. Matsumoto, T. Sakaguchi, H. Kimura, M. Doi, K. Minagawa, Y. Matsuzawa, and K. Yoshikawa, J. Polym. Sci., Part B: Polym. Phys. 30, 779 (1992).

${ }^{25}$ L. D. Landau and E. M. Lifshitz, Statistical Physics (Pergamon, London, 1958).

${ }^{26}$ T. Sakaue and K. Yoshikawa, J. Chem. Phys. 117, 6323 (2002).

${ }^{27}$ N. Yoshinaga, Prog. Theor. Phys. Suppl. 161, 397 (2006).

${ }^{28}$ M. Muthukumar, Philos. Trans. R. Soc. London, Ser. A 361, 539 (2003).

${ }^{29}$ W. Hu, D. Frenkel, and V. B. F. Mathot, Macromolecules 36, 8178 (2003). 